\title{
Interbed demultiple using Marchenko redatuming on 3D field data of the Santos basin
}

\author{
Myrna Staring and Kees Wapenaar (Delft University of Technology)
}

\begin{abstract}
Copyright 2019, SBGf - Sociedade Brazileira de Geofísica
This paper was prepared for presentation during the $16^{\text {th }}$ International Congress of the Brazilian Geophysical Society held in Rio de Janeiro, Brazil, 19-22 August, 2019.

Contents of this paper were reviewed by the Technical Committee of the $16^{\text {th }}$ International Congress of the Brazilian Geophysical Society and do not necessarily represent any position of the SBGf, its officers or members. Electronic reproduction or storage of any part of this paper for commercial purposes without the written consent of the Brazilian Geophysical Society is prohibited.
\end{abstract}

\begin{abstract}
We apply Marchenko redatuming using an adaptive double-focusing method to 3D field data of the Santos basin, Brazil. This method was already successfully applied to 2D field data and we now study the acquisition geometry and preprocessing requirements in 3D. We start from 3D synthetic data modeled on a dense grid of colocated sources and receivers and decimate down to a realistic NAZ streamer acquisition. The synthetic tests show that the sail line spacing and the missing outer cables are the acquisition parameters with the strongest effect on Marchenko redatuming. We can interpolate for the sail line spacing and the near offsets, but the missing outer cables are unfortunately a limitation of the acquisition. After applying the proposed interpolation to $3 \mathrm{D}$ field data, interbed multiples are successfully predicted and subtracted from the target area, resulting in a significant improvement in the geological interpretation. Naturally, the pre-processing requirements and challenges strongly depend on the acquisition geometry and the geology of the area under investigation (e.g. water depth, shape of the overburden, maximum dip). Hence, these tests only give a general idea about the limitations of 3D Marchenko redatuming.
\end{abstract}

\section{Introduction}

The presence of interbed multiples in seismic reflection data is a long-standing problem in Geophysics. First reports date back to 1948, when Sloat (1948) gave a detailed description of this phenomenon. Since then, many methods have been proposed. From methods that transform the data to another domain in which primaries and multiples separate (e.g. Hampson (1986), Foster and Mosher (1992)), to more specialized methods that convolve and correlate the data with itself to reconstruct interbed multiples from the primary reflections (e.g. Weglein et al. (1997), Jakubowicz (1998)). The Marchenko method belongs to the convolution and correlation-type methods, but stands out due to its straight-forward and data-driven approach. Using the reflection response and a smooth velocity model, we can retrieve primaries and all orders of interbed multiples at any desired depth level (Broggini et al. (2012); Wapenaar et al. (2013)). The overlying layers do not need to be resolved first, and it is also not necessary to identify multiple generators.
In a previous paper (Staring et al. (2018)), we reported on the successful application of Marchenko redatuming to 2D field data of the Santos basin. A clear prediction and removal of interbed multiples could be observed, which significantly improved the geological interpretation in the target area. Naturally, the next step is testing multiple prediction and removal on 3D field data. In order to successfully apply Marchenko redatuming to 3D field data, the acquisition and preprocessing requirements have to be clear. Tests on 2D synthetic data and field data have shown that a reflection response without direct wave, ghosts, surface-related multiple, source signature and noise is needed as input for Marchenko redatuming. In addition, a dense grid of sources and receivers is required. However, we have not yet considered the 3D aspects and the limitations of conventional streamer data. Streamer data misses the negative offsets, the near offsets and usually has a limited crossline aperture. Therefore, we wish to test these effects on 3D synthetic data before starting with the field data application.

Since the 2D field data application of Marchenko redatuming to the Santos basin was successful, we continue using data from this region. It is often used to test multiple prediction and subtraction methods (Griffiths et al. (2011), Pereira et al. (2018), Krueger et al. (2018)), since it has a highly reflective salt structure that generates strong interbed multiples (Cypriano et al. (2015)). These multiples interfere with the primaries of the pre-salt reservoir, thereby causing artefacts in its image. The interbed multiples can be clearly distinguished from the primaries, such that the performance of the multiple prediction and removal methods can be accurately evaluated.

\section{Marchenko redatuming by adaptive double-focusing}

Double-focusing is a two-step process (Figure 1). The first step uses the single-focusing Marchenko method to create virtual receivers at the redatuming level. The Marchenko method retrieves so-called focusing functions. When injecting these functions into the medium, they focus at the desired focal point and act either as a virtual receiver that measures a Green's function or as a virtual source that emits a Green's function (Wapenaar et al. (2013)). These Green's functions contain all primaries and all orders of interbed multiples. The retrieval of the upgoing Green's function $\hat{G}^{-}$and the downgoing focusing function $\hat{f}^{+}$can be written as a series (van der Neut et al. (2015b)):

$$
\hat{G}^{-}\left(\boldsymbol{x}_{F}, \boldsymbol{x}_{S}, t\right)=\sum_{i=0}^{\infty} \hat{G}_{i}^{-}\left(\boldsymbol{x}_{F}, \boldsymbol{x}_{S}, t\right)=\Psi \mathcal{R} \sum_{i=0}^{\infty} \Omega^{i} \hat{f}_{0}^{+}\left(\boldsymbol{x}_{S}, \boldsymbol{x}_{F}, t\right)
$$

and 

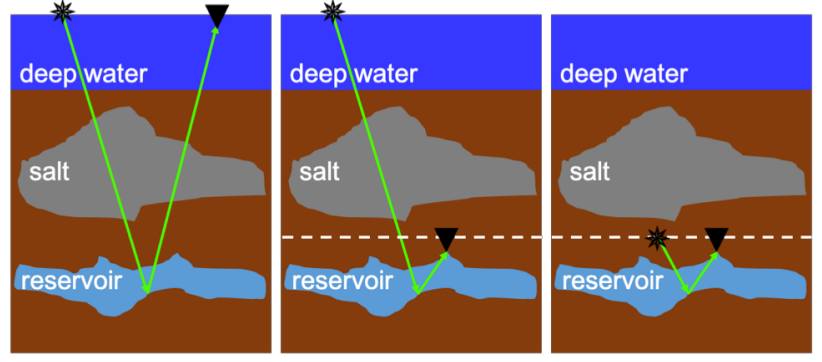

The reflection response

Figure 1 - Illustration of left) the reflection response, middle) single-focusing, and right) double-focusing.

$\hat{f}^{+}\left(\boldsymbol{x}_{S}, \boldsymbol{x}_{F}^{\prime}, t\right)=\sum_{j=0}^{\infty} \hat{f}_{j}^{+}\left(\boldsymbol{x}_{S}, \boldsymbol{x}_{F}^{\prime}, t\right)=\sum_{j=0}^{\infty} \Omega^{j} \hat{f}_{0}^{+}\left(\boldsymbol{x}_{S}, \boldsymbol{x}_{F}^{\prime}, t\right)(2)$

Here $\hat{G}_{i}^{-}$and $\hat{f}_{j}^{+}$denote individual updates of the upgoing Green's function and the downgoing focusing function. $\boldsymbol{x}_{S}$ and $\boldsymbol{x}_{F}$ represent a grid of sources at the acquisition surface and a grid of focal points (virtual receivers) at the redatuming level respectively. The direct downgoing focusing function $\hat{f}_{0}^{+}$is used to initiate the retrieval of both the upgoing Green's function and the downgoing focusing function. We model this wavefield in a smooth velocity model using an Eikonal solver. The $\wedge$ symbol represents a band-limitation, which we obtain by convolving the modeled direct wave $\hat{f}_{0}^{+}$with a zero-phase wavelet covering the frequency content of the data. The symbol $\Omega=\theta R^{*} \theta R$ indicates a series of operations. First, it convolves the reflection response $\mathrm{R}$ with the direct downgoing focusing function $\hat{f}_{0}^{+}$. Next, time-window $\theta$ is applied. This window separates the focusing function and the Green's function in time, using the causality assumption that the focusing function focuses before it acts as either a virtual source or a virtual receiver. Applying the window $\theta$ results in the focusing function, whereas the window $\Psi=\mathrm{I}-\theta$ results in the Green's function. After the windowing step, the result is correlated with the reflection response. After that, it is windowed again. Depending on the number of iterations ( $i$ and $j$ ), operator $\Omega$ is applied multiple times, thus convolving and correlating the data with itself to construct the interbed multiples.

The reflection response is receiver-redatumed after singlefocusing. Next, we convolve the downgoing focusing function at virtual sources with the upgoing Green's function at virtual receivers (Wapenaar et al. (2016), van der Neut et al. (2018)):

$\hat{\hat{G}}^{-+}\left(\boldsymbol{x}_{F}, \boldsymbol{x}_{F}^{\prime}, t\right)=\int_{\partial \mathbb{D}_{0}} \hat{G}^{-}\left(\boldsymbol{x}_{F}, \boldsymbol{x}_{S}, t\right) * \hat{f}^{+}\left(\boldsymbol{x}_{S}, \boldsymbol{x}_{F}^{\prime}, t\right) d^{2} \boldsymbol{x}_{S}$

This operation results in downwards radiating virtual sources and virtual receivers that measure the upgoing wavefields. $\boldsymbol{x}_{F}$ and $\boldsymbol{x}_{F}^{\prime}$ are grids of virtual sources and receivers at the redatuming level. The integral over the acquisition surface $\partial \mathbb{D}_{0}$ allows us to parallelize the doublefocusing method over pairs of focal points, which is especially useful when handling large volumes of $3 \mathrm{D}$ field data.
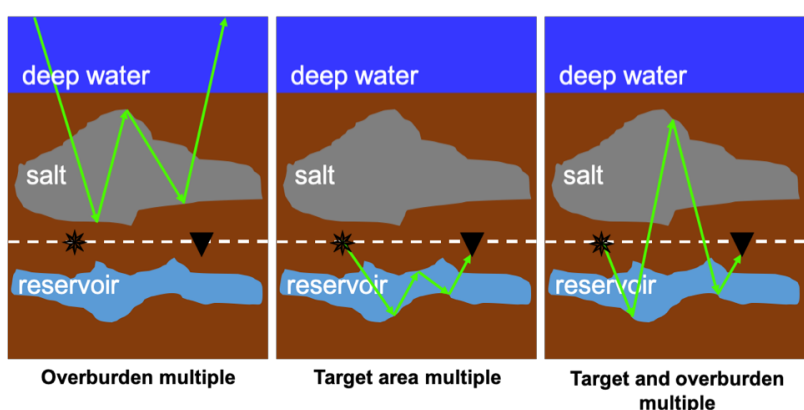

Figure 2 - Illustration of different types of interbed multiples left) multiple generated in the overburden, middle) multiple generated in the target area, and right) multiple generated by interactions between the target and the overburden.

Figure 2 shows examples of different types of interbed multiples. Interbed multiples generated in the overburden are not part of the redatumed response. We redatumed in the physical medium, so interactions between the target zone and the overburden remain. In our case, these remaining interactions arrive significantly later than the primaries of the target area and do not cause artefacts in the image. However, this could be a problem in other geological settings. Also, we do not remove interbed multiples generated in the target area.

When studying the series in equations 1 and 2, a useful property can be observed. The first estimates of both wavefields, $\hat{G}_{0}^{-}$and $\hat{f}_{0}^{+}$, contain primaries and all orders of interbed multiples. Updates of these terms $\left(\widehat{G}_{1}^{-}, \hat{G}_{2}^{-}, \hat{G}_{3}^{-}\right.$, etc. and $\hat{f}_{1}^{+}, \hat{f}_{2}^{+}$nd $\hat{f}_{3}^{+}$, etc.) contain predictions of interbed multiples. The first updates, $\hat{G}_{1}^{-}$and $\hat{f}_{1}^{+}$, contain the predictions of first-order interbed multiples with incorrect amplitudes. Next updates correct for the amplitudes of the first-order multiples and predict higher-order multiples. The geological setting of the Santos basin has 3 main multiple generators: the water bottom, the top of salt and the bottom of salt. Looking at this geometry (see Figure 3), it will mainly be the first-order interbed multiples that create artefacts in the target area. Therefore, we will only need the first updates $\hat{G}_{1}^{-}$and $\hat{f}_{1}^{+}$to predict these multiples. When inserting the series of equations 1 and 2 into equation 3 , while only keeping the initial estimates and the

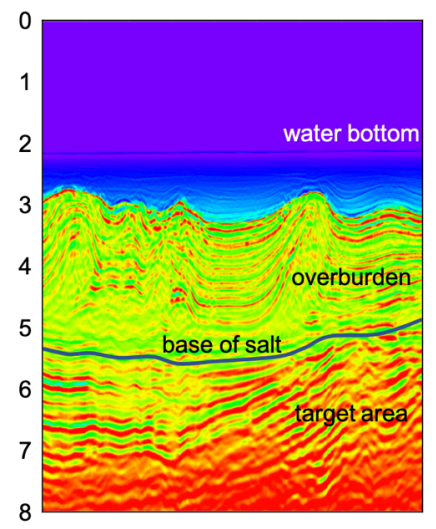

Figure $3-2 D$ slice of the Santos basin velocity model. 
first updates, we obtain the following series for doublefocusing:

$$
\begin{aligned}
& \hat{G}^{-+}\left(\boldsymbol{x}_{F}, \boldsymbol{x}_{F}^{\prime}, t\right)= \\
& \sum_{i=0}^{\infty} \sum_{j=0}^{\infty} \int_{\partial \mathbb{D}_{0}} \hat{G}_{i}^{-}\left(\boldsymbol{x}_{F}, \boldsymbol{x}_{S}, t\right) * \hat{f}_{j}^{+}\left(\boldsymbol{x}_{S}, \boldsymbol{x}_{F}^{\prime}, t\right) d^{2} \boldsymbol{x}_{S} \\
& \approx \int_{\partial \mathbb{D}_{0}} \hat{G}_{0}^{-}\left(\boldsymbol{x}_{F}, \boldsymbol{x}_{S}, t\right) * \hat{f}_{0}^{+}\left(\boldsymbol{x}_{S}, \boldsymbol{x}_{F}^{\prime}, t\right) d^{2} \boldsymbol{x}_{S} \\
& +\int_{\partial \mathbb{D}_{0}} \hat{G}_{1}^{-}\left(\boldsymbol{x}_{F}, \boldsymbol{x}_{S}, t\right) * \hat{f}_{0}^{+}\left(\boldsymbol{x}_{S}, \boldsymbol{x}_{F}^{\prime}, t\right) d^{2} \boldsymbol{x}_{S} \\
& +\int_{\partial \mathbb{D}_{0}} \hat{G}_{0}^{-}\left(\boldsymbol{x}_{F}, \boldsymbol{x}_{S}, t\right) * \hat{f}_{1}^{+}\left(\boldsymbol{x}_{S}, \boldsymbol{x}_{F}^{\prime}, t\right) d^{2} \boldsymbol{x}_{S} .
\end{aligned}
$$

Here the first term of the approximation, $\hat{G}_{0}^{-} \hat{f}_{0}^{+}$, contains the redatumed response that has both primaries and interbed multiples. The second term $\hat{G}_{1}^{-} \hat{f}_{0}^{+}$has first-order sourceside interbed predictions with opposite polarity, while the third term $\hat{G}_{0}^{-} \hat{f}_{1}^{+}$contains first-order receiver-side multiples with opposite polarity. We have left out the fourth term $\hat{G}_{1}^{-} \hat{f}_{1}^{+}$that contains first-order source-and-receiver side interbed predictions. In our case, this term did not contain any new events. However, note that the approximation in equation 4 can be extended to also include higher-order terms depending on the needs of the dataset. We use an adaptive filter in the curvelet domain (e.g., Wu and Hung (2015)) to compensate for the missing amplitude updates that would otherwise be provided by higher-order terms.

\section{D synthetic tests}

We performed a series of $3 \mathrm{D}$ synthetic tests to determine the correct preprocessing of the 3D field data. To model our synthetics, we used the result of an acoustic inversion of the field data, such that the contrasts realistically represent the geology of the Santos basin. We start with a sail line spacing of $75 \mathrm{~m}$, having inline offsets ranging from 0 to $10 \mathrm{~km}$ and a crossline aperture of $1800 \mathrm{~m}$. Using the adaptive double-focusing method, we redatum to a $25 \mathrm{~m}$ by $37.5 \mathrm{~m}$ grid of focal points above the base of salt. Figure 4 shows the result. On the left is the RTM image of the reflection response, zoomed in at the target area. Interbed multiples clearly cause artefacts in the image. The middle image shows an RTM image of $\hat{G}_{0}^{-} \hat{f}_{0}^{+}$, the first term of adaptive double-focusing. This term is the redatumed response that contains both primaries and interbed multiples. Note that this image is constructed from redatumed data, while the left image is constructed from data at the acquisition surface. Nevertheless, the two images should be comparable. The result of adaptive double-focusing is on the right in Figure 4, displaying a successful multiple prediction and removal according to equation 4. Note the white dashes and arrows, they indicate locations where multiple removal is visible. Below the inline images are depth slices at $5900 \mathrm{~m}$ depth. The red arrows and half-circles indicate the multiples that are being removed. This result is promising and proves that our 3D implementation works on a dense acquisition geometry, but it is not a guarantee that it will work on more sparsely sampled data.
Therefore, we decimated the acquisition geometry to observe the effect on our method. Figure 5 shows the RTM images of the adaptive double-focusing result for different sail line spacing's. Decimating from $75 \mathrm{~m}$ sail line spacing to $150 \mathrm{~m}$ sail line spacing does not have a visible effect, but deterioration is clearly visible when using a sail line spacing of $300 \mathrm{~m}$ and $450 \mathrm{~m}$. Artefacts due to multiples are visible in the image of the target where the adaptive double-focusing method suffers to construct and subtract the interbed multiples. Thus, we conclude that the field data should be interpolated to a sail line spacing of $150 \mathrm{~m}$ for the adaptive double-focusing method to be successful in our geological setting. Note that the tests in Figure 5 will be different in another setting. This is merely to illustrate our process of testing to determine the correct preprocessing for the field data. Preprocessing requirements and challenges strongly depend on the acquisition geometry and the geology of the area under investigation (e.g. water depth, shape of the overburden, maximum dip).

Additional tests (that are not shown in this paper) evaluate the effect of removing the negative offsets, the near offsets $(0-250 \mathrm{~m})$, the far offsets $(6250-10000 \mathrm{~m})$ and the outer cables (decrease crossline aperture from $1800 \mathrm{~m}$ to 900 $\mathrm{m})$. These tests show that the negative offsets can be reconstructed using source-receiver reciprocity (Wapenaar et al. (2004)). The near offsets surprisingly do not pose a significant problem, which can possibly be explained by the fact that we have a very deep target area (see Figure 3 ). Although the effect is not strong, we will interpolate for the near offsets on the field data. The removal of the far offsets also did not cause a significant difference. This is a promising result, since we cannot interpolate for this parameter. Lastly, the removal of the outer cables had a significant effect, although a fair amount of primaries and multiples could still be constructed. From these tests, we conclude that source-receiver reciprocity should be applied to our 3D field data, in addition to near offset interpolation and sail line interpolation. The outer cables are unfortunately a limitation of the acquisition that we cannot interpolate for. These conclusions only pertain to this specific dataset, and cannot be applied to other datasets. However, a general conclusion is that the $3 D$ adaptive Marchenko method appears to be quite robust, which is promising for the field data application.

As a final synthetic test, we model synthetic data using the field data geometry, where the acquisition consisted of 6 streamers with 150 cable spacing and $450 \mathrm{~m}$ sail line spacing. The cable length is $6000 \mathrm{~m}$, with offsets from 250 $\mathrm{m}$ to $6250 \mathrm{~m}$. The inline shot and receiver spacing is $50 \mathrm{~m}$. The aperture in the crossline direction is $900 \mathrm{~m}$. We interpolate according to the conclusions of our decimation tests. Figure 6 shows the result of predicting and adaptively subtracting the interbed multiples according to equation 4 . The result shows the correct prediction and removal of the interbed multiples compared to the redatumed response in the middle. However, note that the RTM image of the reflection response is different from the RTM image of $\hat{G}_{0}^{-} \hat{f}_{0}^{+}$. These images should be comparable, but they are not. The difference appears to be due to the coarse sampling and the finite aperture, since the images in Figure 4 are much more comparable. We interpolated the data 


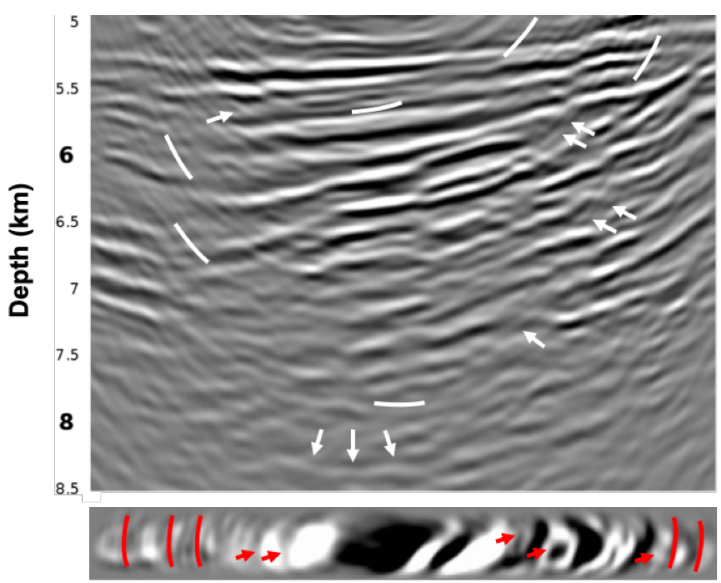

Reflection response

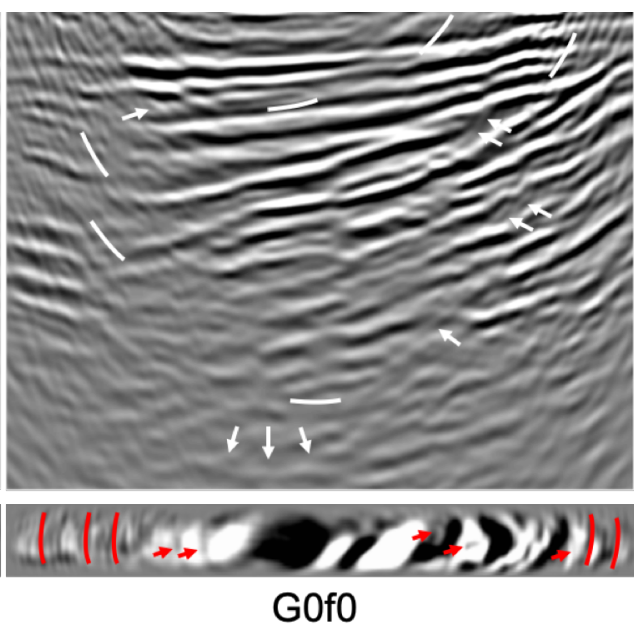

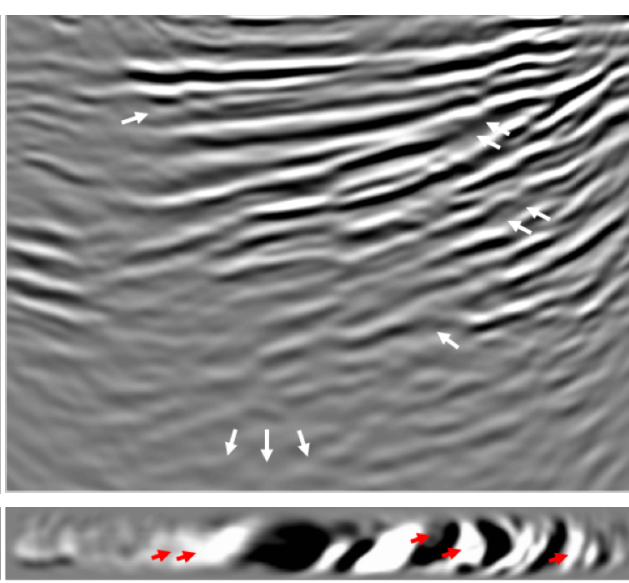

Result adaptive double-focusing

Figure 4 - RTM images of the result from the adaptive double-focusing method on $3 D$ synthetic data with a dense acquisition geometry. The depth slices were taken at $5900 \mathrm{~m}$ depth. Left) RTM image of the reflection response, zoomed in at the target area. Middle) $R T M$ image of $\widehat{G}_{0}^{-} \hat{\boldsymbol{f}}_{\mathbf{0}}^{+}$, the redatumed response containing both primaries and interbed multiples. This term is comparable to the $R T M$ of the reflection response. Right) the result of adaptive double-focusing after adaptively subtracting the multiple predictions.
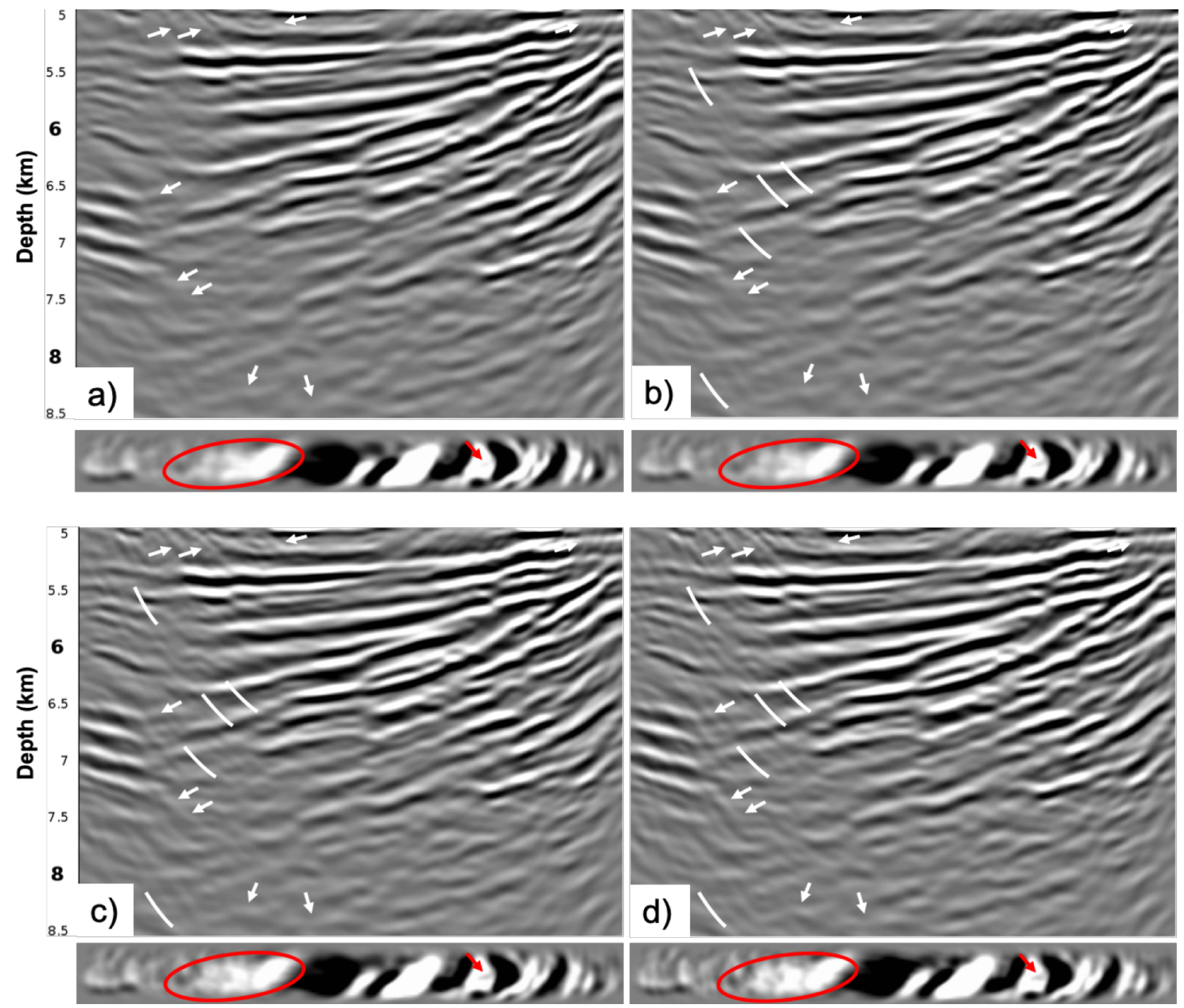

Figure 5 - RTM images of tests with different sail line spacing. All images are the result of the adaptive double-focusing method, thus after multiple prediction and subtraction. The depth slices were taken at $5900 \mathrm{~m}$ depth. a) $75 \mathrm{~m}$ sail line spacing, b) $150 \mathrm{~m}$ sail line spacing, c) $300 \mathrm{~m}$ sail line spacing, and d) $450 \mathrm{~m}$ sail line spacing. 
and back-propagated it with a convolution in equation 1 , instead of using a finite-difference method (which the RTM method applies to the reflection response at the acquisition surface). When the data is finely sampled and has sufficient aperture, back-propagation with a convolution should be equivalent to back-propagation using a finitedifference method, as shown in Figure 4. However, the equivalence is lost when the data is sparsely sampled, as we see in Figure 6. Therefore, we will have to put much care in the interpolation for the field data.

\section{D field data}

Finally, we continue with the field data application. Preprocessing included designature and deghosting. Also, we removed surface-related multiples, the evanescent wavefield and noise. Shot and receiver positions were regularized and interpolation for the near offsets and the sail line spacing was applied. In addition, we reconstructed the missing negative offsets using source-receiver reciprocity.

Figure 7 shows the result of applying the adaptive doublefocusing method to $3 \mathrm{D}$ field data. A significant improvement is visible in the image. The method does well at constructing both the primaries and the interbed multiples, and also seems to be able to subtract the predictions without significant damage to the primaries. Similar to Figure 6, we observe a difference between the RTM image of the reflection response and the RTM image of the redatumed response. A difference in illumination on the right side of the image (see the white arrows) indicates this most clearly. However, this difference is far less pronounced than in Figure 6, which indicates that the interpolation for the sail line spacing and the near offsets has been sufficient to perform a convolution for backpropagation.

\section{Conclusion}

We successfully applied the adaptive double-focusing method on 3D field data of the Santos basin. Decimation tests on realistic synthetic data show that the method is most sensitive to the sail line spacing and the missing outer cables. The missing negative offsets can be reconstructed using source-receiver reciprocity. Surprisingly, the removal of the near and the far offsets had little effect on the result. Based on these results, we performed source-receiver reciprocity to reconstruct the negative offsets and interpolation for the near offsets and the sail line spacing to 3D synthetic data modeled on a field data geometry. The adaptive double-focusing method predicted and subtracted interbed multiples, but a difference between the RTM image of the reflection response at the acquisition surface and the RTM image of the redatumed response indicates that the interpolation was insufficient. The data needs to be finely sampled and have sufficient aperture to make backpropagation with a convolution comparable to finitedifference modeling. Therefore, much care has to be put into interpolation for the field data application.

We performed interpolation for the near offsets and the sail line spacing with much care, and the result shows that the difference between the RTM image of the reflection response and the RTM image of the redatumed response is indeed less. The adaptive double-focusing method correctly predicted and subtracted the interbed multiples, resulting in an improvement in the geological interpretation.

We remark that these results are only valid for our particular dataset. These tests should be repeated for different geological settings, where other parameters might play a role depending on the depth of the target area and the geological dip in the crossline direction. These results are very promising and indicate a robustness of the adaptive double-focusing method for sparse acquisition geometries when interpolation is carried out sufficiently.

Due to the fact that the RTM image of the reflection response and the RTM image of the redatumed response are not completely comparable, it is difficult to quality check our method. In addition, we can only perform a quality check after migration and not in the redatumed domain. Therefore, we propose to look into other Marchenko-type methods that allow for an easier quality check. For example, van der Neut and Wapenaar (2016) propose an adaptive overburden elimination method that does not require redatuming, such that the predicted interbed multiples can be subtracted directly from the reflection response. In addition, Meles et al. (2016) propose a method that only predicts primaries, thereby terminating the need for multiple prediction and removal.

\section{Acknowledgments}

This research was performed in the framework of the project "Marchenko imaging and monitoring of geophysical reflection data", which is part of the Dutch Open Technology Programme with project number 13939, and financially supported by NWO Domain Applied and Engineering Sciences. The research of Kees Wapenaar has received funding from the European Research Council (ERC) under the European Union's Horizon 2020 research and innovation programme (grant agreement No: 742703). We thank Roberto Pereira and Adel Khalil from CGG Rio de Janeiro and CGG Den Haag for a fruitful collaboration.

\section{References}

BROGGINI, F., R. SNIEDER, \& K. WAPENAAR, 2012, Focusing the wavefield inside an unknown 1d medium: Beyond seismic interferometry: Geophysics, 77, A25-A28.

CYPRIANO, L., F. MARPEAU, R. BRASIL, G. WELTER, H. PRIGENT, H. DOUMA, M. VELASQUES, J. BOECHAT, P. DE CARVALHO, C. GUERRA, et al., 2015, The impact of inter-bed multiple attenuation on the imaging of pre-salt targets in the Santos basin off-shore Brazil: Presented at the 77th EAGE Conference and Exhibition 2015.

FOSTER, D. J. \& C. C. MOSHER, 1992, Suppression of multiple reflections using the Radon transform: Geophysics, 57, 386-395.

GRIFFITHS, M., J. HEMBD \& H. PRIGENT, 2011, Applications of interbed multiple attenuation: The Leading Edge, 30, 906-912.

HAMPSON, D., 1986, Inverse velocity stacking for multiple elimination, in SEG Technical Program Expanded Abstracts 1986, 422-424, Society of Exploration Geophysicists.

JACUBOWICZ, H., 1998, Wave equation prediction and removal of interbed multiples, in SEG Technical Program Expanded Abstracts 1998 1527-1530, Society of Exploration Geophysicists.

KRUEGER, J., D. DONNO, R. PEREIRA, D. MONDINI, A. SOUZA, J. ESPINOZA \& A. KHALIL, 2018, Internal multiple attenuation for four presalt fields in the Santos basin, Brazil, in SEG Technical Program Expanded Abstracts 2018, 4523-4527, Society of Exploration Geophysicists. 
MELES, G. A., K. WAPENAAR \& A. CURTIS, 2016, Reconstructing the primary reflections in seismic data by Marchenko redatuming and convolutional interferometry: Geophysics, 81, Q15-Q26.

PEREIRA, R., D. MONDINI, and D. DONNO, 2018, Efficient 3d internal multiple attenuation in the Santos basin: Presented at the 80th EAGE Conference and Exhibition 2018

SLOAT, J., 1948, Identification of echo reflections: Geophysics, 13, 2735.

STARING, M., R. PEREIRA, H. DOUMA, J. VAN DER NEUT, and K. WAPENAAR, 2018, Source-receiver Marchenko redatuming on field data using an adaptive double-focusing method: Geophysics, 83, S579-S590.

VAN DER NEUT, J., K. WAPENAAR, J. THORBECKE, E. SLOB \& I. VASCONCELOS, 2015b, An illustration of adaptive Marchenko imaging: The Leading Edge, 34, 818-822.

VAN DER NEUT, J. and K. WAPENAAR, 2016, Adaptive overburden elimination with the multidimensional Marchenko equation: Geophysics, 81, T265-T284.

VAN DER NEUT, J., J. BRACKENHOFF, M. STARING, L. ZHANG, S. DE RIDDER, E. SLOB, and K. WAPENAAR, 2018, Single-and doublesided marchenko imaging conditions in acoustic media: IEEE Transactions on Computational Imaging, 4, 160-171.
WANG, P., S. RAY, C. PENG, Y. LI, and G. POOLE, 2013, Premigration deghosting for marine streamer data using a bootstrap approach in tau-p domain, in SEG Technical Program Expanded Abstracts 2013, 42214225, Society of Exploration Geophysicists.

WAPENAAR, K., F. BROGGINI, E. SLOB, and R. SNIEDER, 2013, Three-dimensional single-sided

Marchenko inverse scattering, data-driven focusing, Green's function retrieval, and their mutual relations: Physical Review Letters, 110, 084301.

WAPENAAR, K., J. THORBECKE \& D. DRAGANOV, 2004, Relations between reflection and transmission responses of three-dimensional inhomogeneous media: Geophysical Journal International, 156 (2), 179 194.

WAPENAAR, K., J. VAN DER NEUT, and E. SLOB, 2016, Unified double- and single-sided homogeneous

Green's function representations: Proc. R. Soc. A, 20160162

WEGLEIN, A. B., F. A. GASPAROTTO, P. M. CARVALHO \& R. H. STOLT, 1997, An inverse scattering series method for attenuating multiples in seismic reflection data: Geophysics, 62, 1975-1989.

WU, X. and B. HUNG, 2015, High-fidelity adaptive curvelet domain primary-multiple separation:

first break, 33, 53-59.

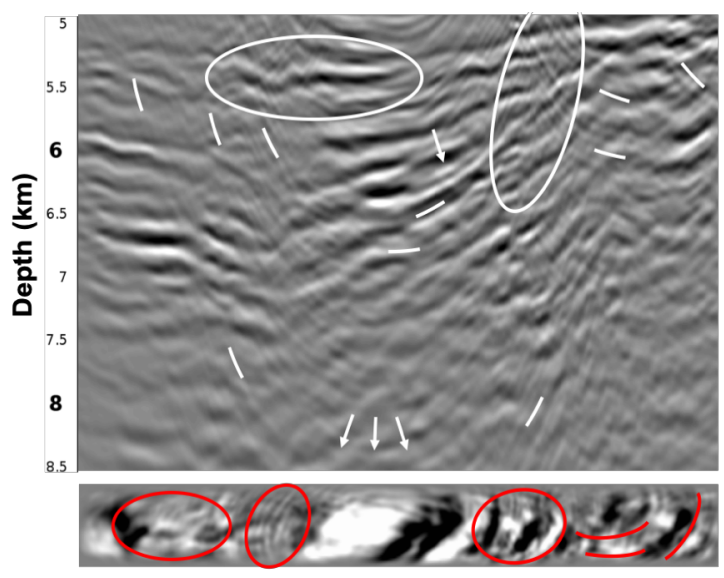

Reflection response

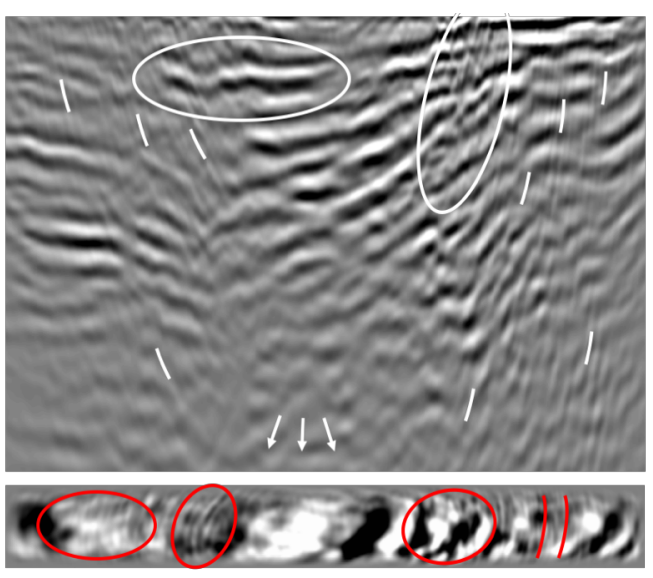

GOfO

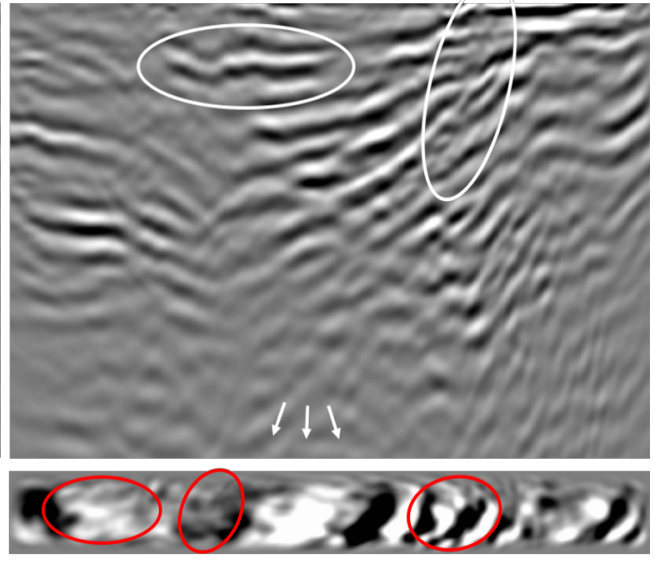

Result adaptive double-focusing

Figure 6 - RTM images of the result from the adaptive double-focusing method on $3 D$ synthetic data modeled on a field data geometry. Interpolation for the near offsets and the sail line spacing was applied. The depth slices were taken at $5900 \mathrm{~m}$ depth. Left) RTM image of the reflection response, zoomed in at the target area. Middle) RTM image of $\widehat{\boldsymbol{G}}_{\mathbf{0}}^{-} \hat{\boldsymbol{f}}_{\mathbf{0}}^{+}$, the redatumed response containing both primaries and interbed multiples. This term is comparable to the RTM of the reflection response. Right) the result of adaptive double-focusing after adaptively subtracting the multiple predictions.
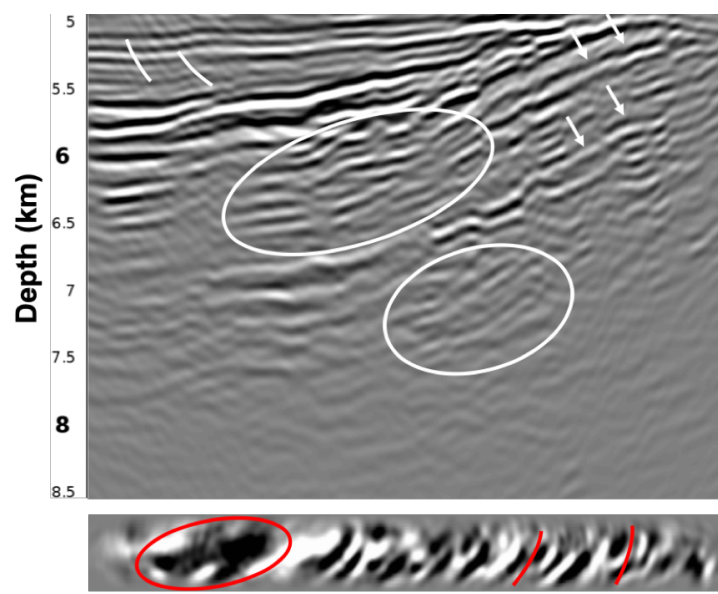

Reflection response
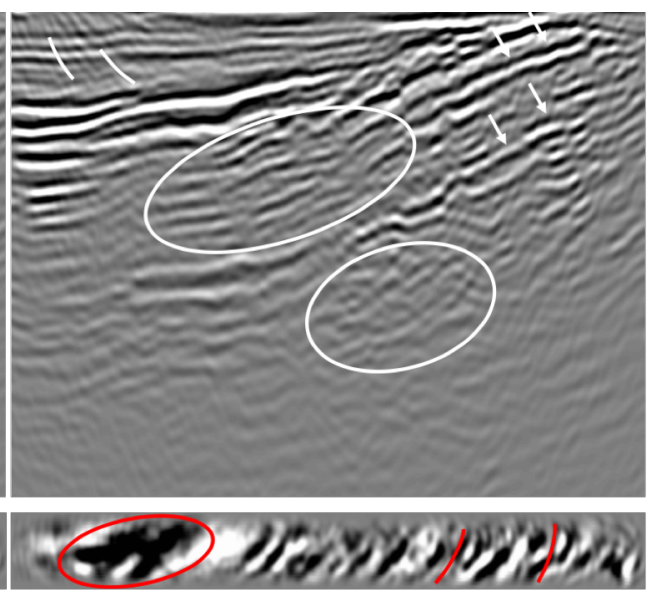

GOfO
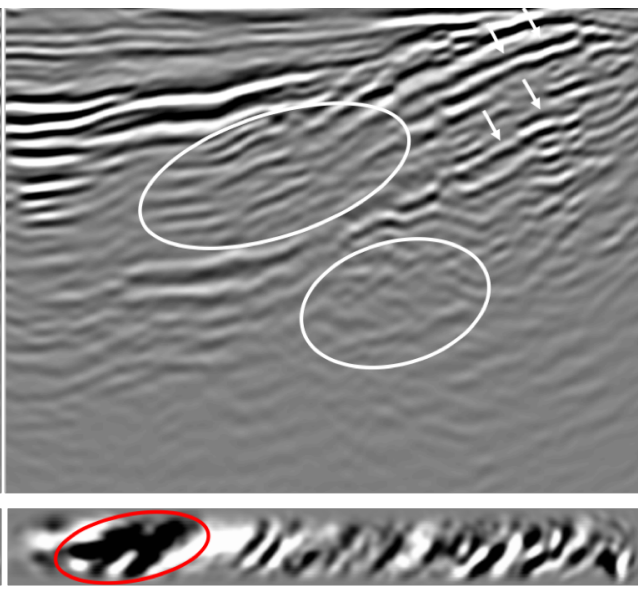

Result adaptive double-focusing

Figure 7 - RTM images of the result from the adaptive double-focusing method on 3D field data. Interpolation for the near offsets and the sail line spacing was applied. The depth slices were taken at $5900 \mathrm{~m}$ depth. Left) RTM image of the reflection response, zoomed in at the target area. Middle) RTM image of $\widehat{G}_{0}^{-} \widehat{f}_{0}^{+}$, the redatumed response containing both primaries and interbed multiples. This term is comparable to the $R T M$ image of the reflection response. Right) the result of adaptive double-focusing after adaptively subtracting the multiple predictions. 